\title{
Non-rigid Surface Tracking for Virtual Fitting System
}

\author{
Naoki Shimizu, Takumi Yoshida, Tomoki Hayashi, Francois de Sorbier and Hideo Saito \\ Graduate School of Science and Technology, Keio University, 3-14-1 Hiyoshi, Kohoku-ku, Yokohama, Japan \\ \{naoki, tkm, tommy, fdesorbi, saito\}@hvrl.ics.keio.ac.jp
}

\begin{abstract}
Keywords: Deformable 3D Registration, Principal Component Analysis, RGB-D Camera, Augmented Reality, Local Feature Point.

Abstract: $\quad$ In this paper, we describe a method for overlaying a texture onto a T-shirt, for improving current virtual fitting system. In such systems, users can try on clothes virtually. In order to realize such a system, a depth camera has been used. These depth cameras can capture 3D data in real time and have been used by some industrial virtual cloth fitting systems. However, these systems roughly, or just do not, consider the shape of the clothes that user is wearing. So the appearance of these virtual fitting systems looks unnaturally. For a better fitting, we need to estimate 3D shape of cloth surface, and overlay a texture of the cloth that the user wants to see onto the surface. There are some methods that register a 3D deformable mesh onto captured depth data of a target surface. Although those registration methods are very accurate, most of them require large amount of processing time or either manually-set markers or special rectangles. The main contribution of our method is to overlay a texture onto a texture of T-shirt in real-time without modifying the surface.
\end{abstract}

\section{INTRODUCTION}

Recent progress in computer vision significantly extended the possibilities of augmented reality, a field that is quickly gaining popularity. Consequently, augmented reality has been applied to many domains like entertainment and navigation.

For clothes retail industry, examples of virtual clothes fitting system, which enables the users to try the clothes on virtually, have been presented. It can be applied to tele-shopping systems over the internet, garment designing, and etc. Recently, commodity RGB-D camera with reasonable price became popular, and practical virtual fitting systems have been released. However, these systems roughly, or just do not, consider the shape of the clothes that user is wearing. So the displayed result of these virtual fitting systems looks unnatural. For a better result, estimation of the shape of cloth surface is required.

We are developing a real-time method for overlaying a texture on a cloth. In our system, a RGB-D camera captures a moving person in front of a display that shows the mirrored input image. Camera and display are mounted such that the user has the impression of looking into a mirror showing the upper part of user's body. A part of the cloth is replaced with a virtual texture, resulting in the user shown to be wearing the virtual cloth in the mirror. For that purpose we developed a dynamic texturing method for non-rigid surface like a T-shirt that registers deformed mesh fit to a surface from RGB and Depth images captured by the camera and renders in real-time a virtual texture onto the moving surface with correct deformation.

Non-rigid surface tracking from a RGB-D camera encounters the problem of surface region detection. In previous methods related to virtual fitting system, special colored shirt or markers were used. For prevailing virtual fitting system, we need to detect surface region from common T-shirt that the user is wearing. For this, we use the texture on the T-shirt to define the target region. However, in most cases, this texture have less feature points, and obviously, are deformed. Most algorithms for feature corresponding points and removing outliers like SIFT (Lowe, 1999) or RANSAC (Chum et al., 2003) are only available for planar surfaces. Therefore, in such situation, the non-rigid surface region should be detected and tracked by using relatively few feature points, and these feature points should be correctly detected from deformed texture. We propose a method based on feature corresponding points on the shirt that can handle these problems.

We improve the method proposed by Hayashi et al. (Hayashi et al., 2012), which can handle non-rigid surface tracking from a few corresponding points. They used manually attached color makers on T-shirt 
to detect the target tracking region. We present a method that can find correct corresponding points even if the target texture is not on planar surface. By using this method, we can remove color makers from the T-shirt. Furthermore, we improved point sampling method, which allowed us to obtain more accurate point cloud from captured 3D data.

One important characteristic of our method is that we achieve accurate non-rigid surface deformation in real-time with simple hardware and equipment. This was achieved by only using inexpensive devices: regular PC, commodity RGB-D camera, and a display. In addition, the users are not required to wear specially prepared T-shirt, such as the ones that are specially colored or have markers attached.

Generally, depth components captured by commodity RGB-D camera contain noises. Therefore, we couldn't get accurate deformed mesh by using 3D reconstruction from these noisy data. Our method is based on learning representative deformation of meshes, deformed mesh is registered to the target surface. The shape of registered mesh is accurate and noise-free.

The rest of this paper is structured as follows. Section 2 briefly describes the related works in non-rigid surface tracking, and states the contribution of this paper. Section 3 describes our system. Section 4 explains the detection of the region on non-rigid object. Section 5 describes the tracking and overlaying texture approach for non-rigid surface in RGB-D sequence. Finally, Section 6 presents experimental results.

\section{RELATED WORKS AND CONTRIBUTION}

Non-rigid surface tracking and texturing have recently been addressed by a number of researchers. Most of them are applying a two or three dimensional deformable model reconstructed from RGB camera.

There are some researches approaching practical virtual fitting system. For example, Hilsmann and Eisert proposed a real-time system that tracks clothes and overlays a texture on them by estimating the elastic deformations of the clothes from a single camera in the 2D image plane (Hilsmann and Eisert, 2009). The problem of self-occlusion is addressed by using a 2D motion model regularizing an optical flow field. It works correctly in real-time, but under an assumption that the motion is small enough, and that the T-shirt is specially colored and textured.

In order to handle larger and sharp deformation, many researches about 3D deformable model recon- struction have been done. Pilet et al. have presented a feature-based fast method which detects and tracks deformable objects in monocular image sequences (Pilet et al., 2007). They applied a wide baseline matching algorithm for finding correspondences. Shen et al. recovered the 3D shape of an inextensible deformable surface from a monocular image sequence (Shen et al., 2010). Their iterative $L_{2}$-norm approximation process computes the non-convex objective function in the optimization. The noise is reduced by applying a $L_{2}$-norm on re-projection errors.

All these 3D reconstruction methods yield impressive results. However, they need sophisticated acquisitions setups or have a high computational cost. We strive for a different goal, i.e. to estimate non-rigid surface of common T-shirt in real-time with normal hardware. For that purpose we are interested in generation of a deformation mode space from the PCA (Principal Component Analysis) of sample triangular meshes (Salzmann et al., 2007). Reducing DOF (Degree of Freedom) by PCA achieved real-time 3D deformable model reconstruction. The non-rigid shape is then expressed by the combination of each deformation mode. This step does not need an estimation of an initial shape or a tracking. Later, they proposed a linear local model for a monocular reconstruction of a deformable surface (Salzmann and Fua, 2011). This method reconstructs an arbitrary deformed shape as long as the homogeneous surface has been learned previously. However, these approaches require a lot of corresponding points between a template image and an input image. Therefore, users have to wear texture-rich T-shirt.

Hayashi et al. improved the method proposed by Salzmann (Hayashi et al., 2012). By using 3D data captured by a RGB-D camera, even though input depth data is noisy, this learning-based method can generate a natural and smooth mesh. And, because the $3 \mathrm{D}$ data is directly captured, texture-rich surface is not required. Furthermore, this method achieves a real-time process by taking the advantage of PCA, a simple method of reducing the dimensionality of the data, which in this case is the meshes. Unfortunately, in this method, users are required to set color markers on their T-shirt for detecting target region.

In our research, we improved non-rigid surface tracking method based on Hayashi's method. The main contribution of this paper lies in a real-time tracking and texture overlaying method for non-rigid surfaces on common T-shirt. Common T-shirt means a T-shirt without rich texture or markers. The difficulty is to detect sufficiently accurate corresponding points from texture on the shirt. We present a method which can detect accurate corresponding 


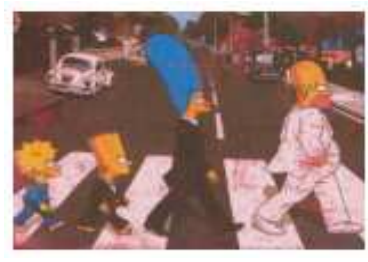

(a) template image

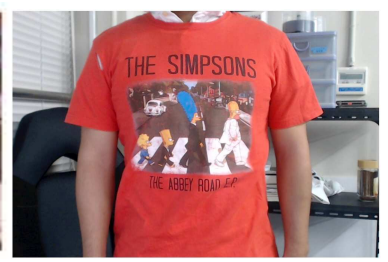

(b) input RGB image
Figure 1: Example of template and input image.

points even if the T-shirt is largely deformed. Along with Hayashi's method, our method can generate a mesh that fits to target surface from at least 7 corresponding points. Additionally, we also used a sophisticated point sampling method. By using this sapling method, even if the positional relationships of detected corresponding points are not uniform, we can get applicable point cloud from input image. As a result, the virtual texture is deformed in real-time and overlaid onto the user's T-shirt.

\section{OVERVIEW}

Our method can work in real-time with a regular PC, and a RGB-D camera (Microsoft Kinect). The user does not need to wear a T-shirt with specific color or markers. However, a certain level of texture on the T-shirt is required.

Before using virtual fitting system, we capture a texture on user's T-shirt without deformation. We call it the "template image". After that, input RGB and Depth images are captured in every frame. Example of template and input image is shown in Figure 1.

In every frame, we need to detect accurate corresponding points between input and template image. However, as shown in Figure 1(b), texture on worn T-shirt is deformed. So if we use matching algorithm based on local features like SIFT (Lowe, 1999), SURF (Bay et al., 2006), many miss-correspondences will occur. For obtaining stable corresponding points, we present local feature detection algorithm suited for deformed texture. Details of this method are described in Section 4.

After detecting corresponding points, we could recognize target non-rigid surface on T-shirt. By using positional relationships of corresponding points, 3D point cloud is sampled from target region. Sampled point cloud are used by non-rigid surface tracking method shown in Section 5. After that, we can get 3D mesh that fits to estimated surface shape. Lastly, by overlaying texture onto this mesh, a virtual texture on the T-shirt is displayed.

\section{KEY POINT DETECTION FROM DEFORMED TEXTURE}

In this section, we describe a method which can detect corresponding points between input and template image, even if input texture is deformed. Conventional local features such as SIFT, SURF, etc., have scale and rotation invariance but often fail to match points when the camera pose significantly changes. In other words, even if camera is stationary, deformation of the texture is assumed to be changes of comparative camera position. Because it also leads to miss-corresponding points. To remove such outliers, RANSAC based algorithms are widely used (Chum et al., 2003). However, the transformation between template and input image is nonlinear, so RANSAC based algorithms do not work well. So we present an accurate corresponding points detection method from deformed input image.

Some local features have scale and rotation invariance resulting from their feature descriptors. Our approach is to collect these invariant features on the generated patterns as seen from different viewpoints. Viewpoint generative learning enables us to train with various data without actually collecting them. As long as we use a local feature to detect or recognize a target, only one template image is needed to learn. Therefore, for offline learning, we generate various patterns, extract stable key points from them and create a database of collected features. After learning, the template image and an input image can be matched by comparing them using the database. The basic idea and detail of this key point detection method is shown in (Yoshida et al., 2013). It is focusing on planar pattern tracking for generic AR application.

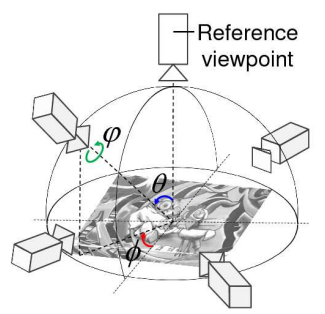

Figure 2: Viewpoint generation model.

\subsection{Generation of Various Patterns}

First, we generate various patterns as seen from different viewpoints that are computed from one template image of the target. We apply perspective transformation. The viewpoint model is shown below in Figure 2. The rotation ranges are set as follows camera longitude $\phi \in\left[-75^{\circ}, 75^{\circ}\right]$, latitude $\theta \in\left[-75^{\circ}, 75^{\circ}\right]$. 
In our method we use rotation invariant features, so that the camera spin $\psi$ becomes constant. To calculate perspective transformation matrix $\mathbf{P}$, we obtain an intrinsic camera parameter matrix $\mathbf{A}$ and an extrinsic parameter, the distance $d$ to a target plane. Moreover, due to scale invariant features, the distance is fixed. Therefore, we have $\mathbf{A}, \mathbf{R}$ and translation matrix $t=(0,0, d)^{T}$ for $\mathbf{P}=\mathbf{A}(\mathbf{R} \mid t)$.

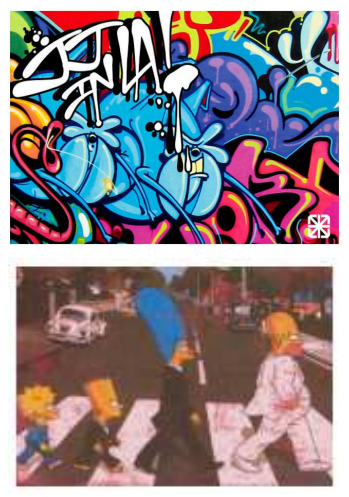

template image
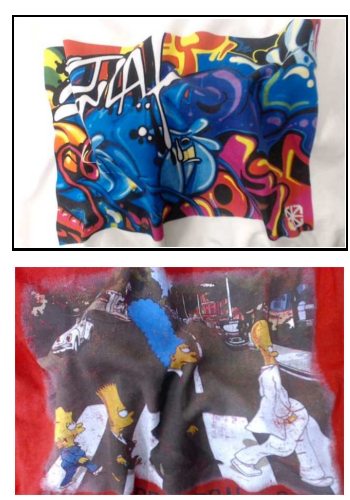

deformed image
Figure 3: Template \& deformed input images.

\subsection{Key Point Detection and Matching}

On each generated pattern, we detect key points by using SIFT (Lowe, 1999). To increase repeatability, we select the stable key points that have high detectability, defined as how often the same key point is detected in different pose patterns, at stable locations. By using these key points generated from various viewpoint, we can get accurate corresponding points between template image and deformed texture in input image.

To confirm effectivity of our approach, we detect corresponding points between 4 deformed textures and template images shown in Figure 3. Table 1 shows our method can detect accurate corresponding points even if the target texture is deformed.

Table 1: Accuracy of corresponding points.

\begin{tabular}{|c|c|c|c|c|}
\hline Template & Method & Correct & Incorrect & Accuracy \\
\hline Upper & SIFT & 74 & 20 & $79 \%$ \\
\hline Upper & Proposed & 22 & 4 & $85 \%$ \\
\hline Lower & SIFT & 145 & 20 & $89 \%$ \\
\hline Lower & Proposed & 29 & 2 & $94 \%$ \\
\hline
\end{tabular}

\section{TEXTURE OVERLAY ONTO NON-RIGID SURFACE}

In this section, we describe our method to overlay a texture onto a non-rigid surface. Figure 4 illus-

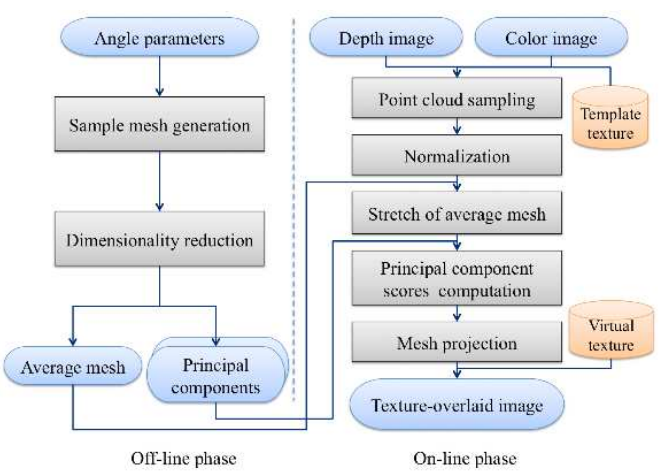

Figure 4: Flow of our method.

trates the flow of our method. Our method is based on Hayashi's method (Hayashi et al., 2012). First, in the off-line phase, we generate deformation models by learning many representative meshes. Because the dimensionality of the mesh in the model is low, we can quickly generate an arbitrarily deformable mesh to fit the target surface in the on-line phase. In addition, this is learning-based approach, so even though the input data is noisy, we can generate a natural mesh that has a smooth shape.

Hayashi's method used color markers to detect target surface region. On the other hand, our method makes it possible to remove such markers because the accurate correspondences from deformed texture are detected by using the method described in Section 4. We also proposed sophisticated point sampling method in Section 5.2.1. By using this sampling method, even if the positional relationships of detected corresponding points are not uniform, we can get suitable point cloud from input sequence.

\subsection{Surface Deformation Models Generation}

In the off-line phase, we generate the deformation models by learning several representative sample meshes. This part is based on Salzmann's method (Salzmann et al., 2007).

In our approach, the target surface and the template texture are rectangular. We introduce a rectangular surface sample mesh made of $m=M \times N$ vertices $\mathbf{V}=\left\{\mathbf{v}_{1}, \ldots, \mathbf{v}_{m}\right\} \subset \mathbb{R}^{3}$.

By using PCA based dimensionality reduction described in (Salzmann et al., 2007), we can get the average mesh $\overline{\mathbf{V}}=\left\{\mathbf{y}_{1}, \ldots, \mathbf{y}_{m}\right\} \subset \mathbb{R}^{3}$ and $N_{c}$ principal component vector $\mathbf{P}_{k}=\left\{\mathbf{p}_{k 1}, \ldots, \mathbf{p}_{k m}\right\} \subset \mathbb{R}^{3}$ which represents some deformation modes. Then an arbitrary mesh can be expressed as follows:

$$
\mathbf{V}=\overline{\mathbf{V}}+\sum_{k=1}^{N_{c}} \omega_{k} \mathbf{P}_{k}
$$


where $\mathbf{V}$ is the vertices of the target surface mesh that we want to generate, $\omega_{k}$ denotes $k^{\text {th }}$ principal component score or weights, and $\mathbf{P}_{k}$ denotes the corresponding principal components or deformation modes. $N_{c}$ is the number of the principal components, which is determined by looking at the contribution rate of the PCA. Once principal component score vector $\Theta=$ $\left\{\omega_{1}, \ldots, \omega_{N_{c}}\right\}$ is known, the surface mesh can be easily reconstructed using Eq. 1.

\subsection{Mesh Registration}

For overlaying virtual texture onto target surface $\mathcal{T}$, we need to estimate the optimal principal component score vector $\Theta$. The principal component score $\omega_{k}$ is described as:

$$
\omega_{k}=(\mathbf{V}-\overline{\mathbf{V}}) \cdot \mathbf{P}_{k}
$$

$\overline{\mathbf{V}}, \mathbf{P}_{k}$ and $\mathbf{V}$ were defined in Eq. 1.

\subsubsection{Point Cloud Sampling}

$\mathbf{V}$ in Eq. 2 is unknown. Thus, the input point cloud of the target surface $\mathcal{T}$ is used as a candidate to replace V. By sampling the input point cloud from $\mathcal{T}$, we can get $\mathbf{V}_{\mathcal{T}}$, which is approximation of $\mathbf{V}$.

Therefore, we sample the point cloud of $\mathcal{T}$ to match its dimensionality to the dimensionality of $\mathbf{V}$. The sampling is done on the input depth image by using corresponding points detected with the method described in Section 4.

Hayashi et al. used linear interpolation for sampling point cloud. It works well only if the corresponding points are located close from the target region's edges. However, in our method, position of detected corresponding points are not ordered in such ways. Because we present a point sampling method based on non-linear transformation.

At first, non-linear transformation matrix is calculated as presented in Eq. 3 and 4.

$$
\begin{gathered}
{\left[\begin{array}{c}
x_{2} \\
y_{2} \\
1
\end{array}\right]=\mathbf{H}\left[\begin{array}{c}
x_{1}^{3} \\
y_{1}^{3} \\
x_{1} \\
y_{1} \\
1
\end{array}\right]} \\
\mathbf{H}=\left[\begin{array}{lllll}
H_{11} & H_{12} & H_{13} & H_{14} & H_{15} \\
H_{21} & H_{22} & H_{23} & H_{24} & H_{25} \\
H_{31} & H_{32} & H_{33} & H_{34} & H_{35}
\end{array}\right]
\end{gathered}
$$

$x_{1}, y_{1}$ denote coordinates of key points in template image, $x_{2}, y_{2}$ denote coordinates of corresponding points in input image. If there are at least 7 corresponding points, we can calculate $\mathbf{H}$. For accurate calculation of $\mathbf{H}$, these corresponding points should
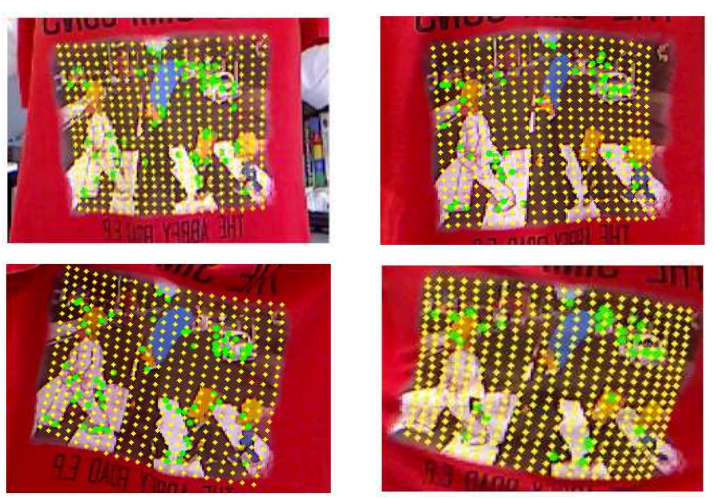

Figure 5: Corresponding points and sampled points. Green points on the surface denote corresponding points. Yellow points are the sampled points.

be spread throughout the target region. By using calculated $\mathbf{H}$, each vertices on $\overline{\mathbf{V}}$ can be transformed to $\mathcal{T}$. These transformed points are used as target sampling point mesh $\mathbf{V}_{\mathcal{T}}$. Figure 5 shows corresponding points and sampled points. Even if the corresponding points are situated discretely, our method can obtain sampled points on the target surface point cloud $\mathcal{T}$.

\subsubsection{Normalization and Stretching Mesh}

Once we get mesh $\mathbf{V}_{\mathcal{T}}$ from the sampled point cloud obtained in Section 5.2.1, we need to transform it to the same coordinate system of $\overline{\mathbf{V}}$ and $\mathbf{P}_{k}$. We used the same rigid transformation matrix $\mathbf{M}$ as described in Hayashi's paper (Hayashi et al., 2012). M transforms data in world coordinate system $\mathbf{V}_{\mathcal{T}}$ to normalized coordinate system which is the same as $\overline{\mathbf{V}}$ and $\mathbf{P}_{k}$.

By using $\mathbf{M}$ transformation, $\mathbf{V}_{\mathcal{T}}$ is transformed to normalized mesh $\mathbf{V}^{\prime}$. We can use $\mathbf{V}^{\prime}$ as approximation of $\mathbf{V}$.

At last, $\overline{\mathbf{V}}$ is stretched so that coordinates of $\overline{\mathbf{V}}$ and $\mathbf{V}^{\prime}$ become similar to each other. In other word, each vertices of $\overline{\mathbf{V}}$ are transformed based on coordinate points. After that, by using these two meshes, we can calculate each principal component score $\omega_{k}$ from Eq. 1.

\subsubsection{Principal Component Scores Computation}

Thus, we can adapt Eq. 1 and Eq. 2 to:

$$
\begin{aligned}
& \omega_{k}=\left(\mathbf{V}^{\prime}-\overline{\mathbf{V}}^{\prime}\right) \cdot \mathbf{P}_{k} . \\
& \mathbf{V}=\overline{\mathbf{V}}^{\prime}+\sum_{k=1}^{N_{c}} \omega_{k} \mathbf{P}_{k} .
\end{aligned}
$$

This means that each $\omega_{k}$ that is calculated in the Eq. 5 is compatible to $\overline{\mathbf{V}}^{\prime}$. Then we get the mesh $\mathbf{V}$ corresponding to $\mathcal{T}$. 

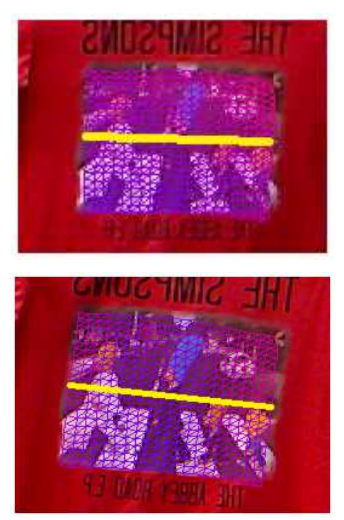

generated mesh

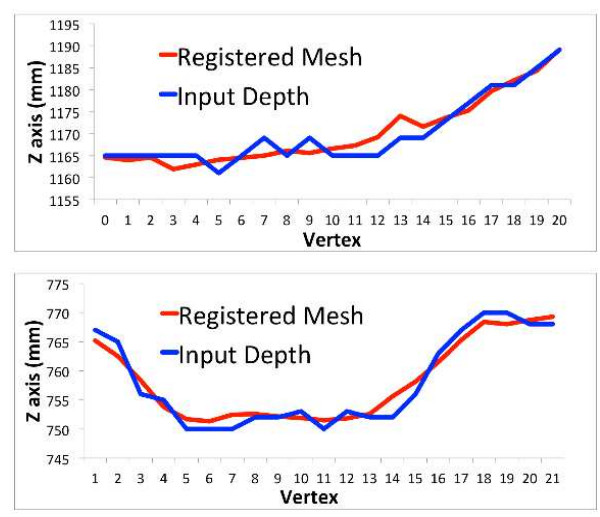

comparison with input depth data

Figure 6: Generated mesh and its comparison with depth data from RGB-D camera. The yellow line of left images denotes the vertices which we used for comparison.

\subsubsection{Mesh Projection}

The last stage is to transform it to the world coordinate system. Because we already know transformation $\mathbf{M}$ from the world coordinate system to the normalized coordinate system, we can transform $\mathbf{V}$ by using $\mathbf{M}^{-1}$.

For the rendering, we define the texture coordinates for each vertex of a surface mesh. Therefore, the virtual texture is overlaid on the target surface obtained by $\mathbf{M}^{-1} \mathbf{V}$.

\section{EXPERIMENTAL RESULTS}

In this section, we make experiments to confirm the effectiveness of our method. All the experiments have been done on a system equipped with a Intel Core i7-3930K 3.20GHz CPU, NVIDIA GeForece GTX $580 \mathrm{GPU}$, and 16.0 GB RAM. We use the RGB-D camera Microsoft Kinect with an image resolution of $640 \times 480$ pixels and a frame rate of $30 \mathrm{fps}$. This RGB-D camera can capture depth component directly by using infrared in real-time. The target surface is a region of a T-shirt with texture. For the resolution of the rectangular mesh, we set both $M$ and $N$ to 21 vertices.

We evaluated generated meshes by comparing depth $(z)$ value between generated mesh and captured from RGB-D camera. The results are shown in Figure 6. Yellow lines on generated mesh denote 20 vertices which we sampled for this evaluation. As Figure 6 shows, the registered mesh is deformed following input depth data. In spite of noisy depth data, our method can generate similar and smooth mesh.

We calculated the processing time because our method is supposed to be used for a real-time virtual fitting application. The result of the average pro- cessing time for 100 frames is shown in Table 2. As a whole, the average processing speed was over 20 frames per second.

Table 2: Processing time.

\begin{tabular}{|c|c|}
\hline Task & Time $(\mathrm{msec})$ \\
\hline Capturing & 11 \\
\hline Key Point Detection & 22 \\
\hline Mesh Registration & 7 \\
\hline Image Rendering & 4 \\
\hline
\end{tabular}

Finally, Figure 7 shows visualization results of the texture overlay onto the non-rigid target surface of 2 kinds of T-shirt. Users can move freely in front of the camera and perform elastic deformations with the cloth, including stretching and bending it. Rotation is possible as long as the texture on the shirt stays visible. Left two columns of Figure 7 demonstrate results using T-shirt with rich texture. Right two columns show results with relatively sparse texture. Even if the T-shirt does not have special color or markers, our method can deform mesh to fit the surface according to the data obtained by RGB-D camera.

\section{CONCLUSIONS AND FUTURE WORKS}

In this paper, we presented a non-rigid surface tracking methodthat registers 3D deformable mesh for texture overlay. Our method has several advantages over the aforementioned previous works. Firstly, our method does not require makers or special color on the target surface. Additionally, registering a deformed mesh is possible with as few as 7 correspondences. This means that our method is available for 

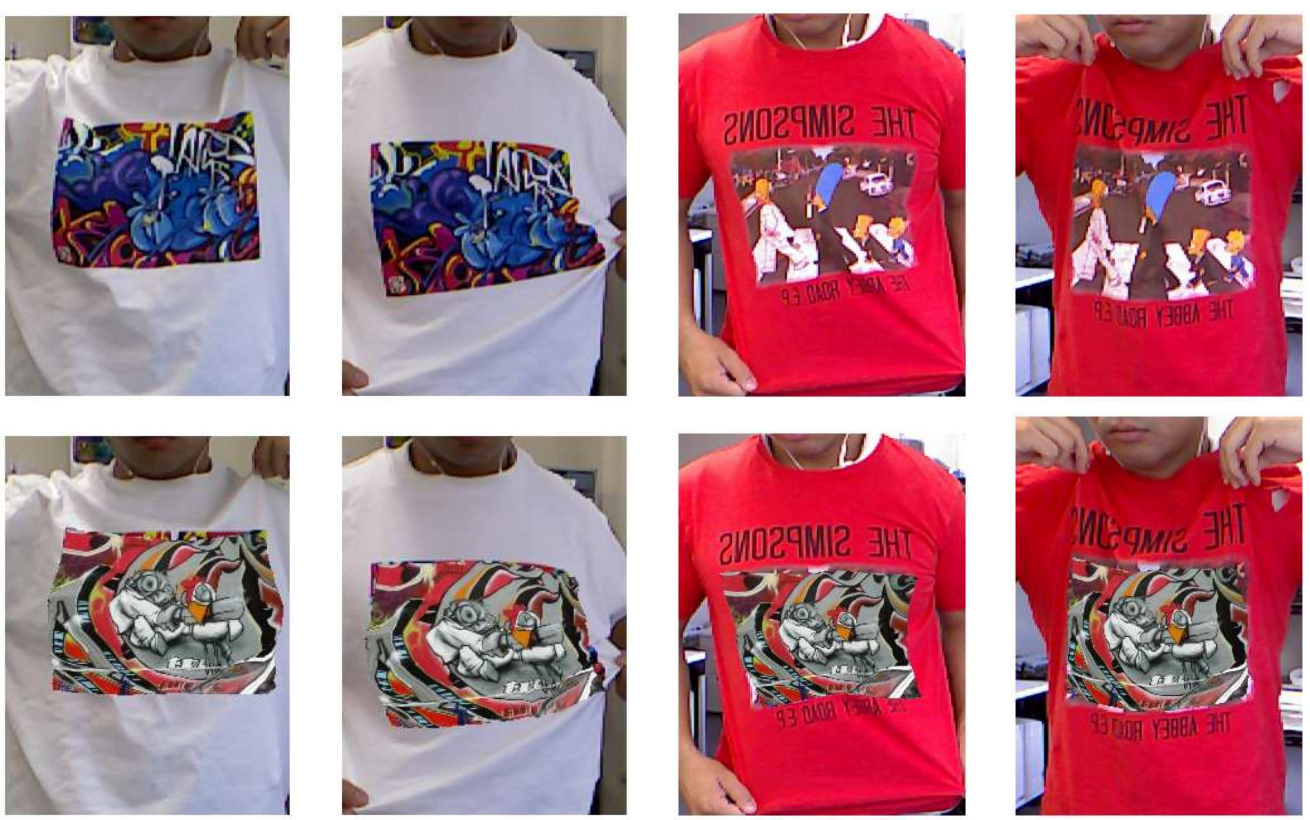

Figure 7: Texture overlaying result. Upper images are input RGB images. Lower images are output images.

relatively poor texture. Secondly, by using low dimension deformable model from PCA, our method obtains a real-time processing.

As a future work, we will improve the method to detect key points from template and input image. The accuracy of registered mesh depends on the positions of key points. If most of the key points are concentrated to a particular region, then the accuracy of the generated mesh becomes lower when it is far from those regions. Therefore we need to improve the key point detection methodto make detecting the key points from wider region possible.

In addition, we will take the light source information into consideration in texturing part for more natural visualization.

\section{REFERENCES}

Bay, H., Tuytelaars, T., and Van Gool, L. (2006). Surf: Speeded up robust features. Computer Vision-ECCV 2006, pages 404-417.

Chum, O., Matas, J., and Kittler, J. (2003). Locally optimized ransac. Pattern Recognition, pages 236-243.

Hayashi, T., de Sorbier, F., and Saito, H. (2012). Texture overlay onto non-rigid surface using commodity depth camera. In VISAPP.

Hilsmann, A. and Eisert, P. (2009). Tracking and retexturing cloth for real-time virtual clothing applications. In Proceedings of the 4th International Conference on Computer Vision/Computer Graphics CollaborationTechniques, pages 94-105. Springer-Verlag.
Lowe, D. (1999). Object recognition from local scaleinvariant features. In Computer Vision, 1999. The Proceedings of the Seventh IEEE International Conference on, volume 2, pages 1150-1157. Ieee.

Pilet, J., Lepetit, V., and Fua, P. (2007). Fast Non-Rigid Surface Detection, Registration and Realistic Augmentation. IJCV , 76(2):109-122.

Salzmann, M. and Fua, P. (2011). Linear local models for monocular reconstruction of deformable surfaces. PAMI, 33(5):931-44.

Salzmann, M., Pilet, J., Ilic, S., and Fua, P. (2007). Surface deformation models for nonrigid 3D shape recovery. PAMI, 29(8):1481-7.

Shen, S., Shi, W., and Liu, Y. (2010). Monocular 3-D tracking of inextensible deformable surfaces under L(2) norm. image processing, 19(2):512-21.

Yoshida, T., Saito, H., Shimizu, M., and Taguchi, A. (2013). Stable keypoint recognition using viewpoint generative learning. In VISAPP. 